\title{
Effect of polybrominated diphenyl ether on development of cultured hippocampal neuron
}

\author{
LIAO ChunYang ${ }^{1}$, DUAN ShuMin ${ }^{2} \&$ JIANG GuiBin ${ }^{1 \dagger}$ \\ ${ }^{1}$ State Key Laboratory of Environmental Chemistry and Ecotoxicology, Research Center for Eco-Environmental Sciences, Chinese \\ Academy of Sciences, Beijing 100085, China \\ ${ }^{2}$ Institute of Neuroscience and Key Laboratory of Neurobiology, Shanghai Institutes for Biological Sciences, Chinese Academy of \\ Sciences, Shanghai 200031, China
}

Polybrominated diphenyl ether (PBDE) is a persistently environmental pollutant ubiquitously found in wildlife and humans. Although concern on PBDE's toxic effects is steadily increasing, its action on the central nervous system (CNS) remains largely unknown. To address this issue, the present study examined the development inhibition of PBDE in neurons. The primary cultured hippocampal neurons of rat were exposed to the commercial decabromodiphenyl ether (deca-BDE), and the neurite length, bifurcation, and synapse formation and maturation were evaluated, based on the confocal microscope imaging. The results showed that the development inhibition in neurons occurred at $15 \mu \mathrm{mol} / \mathrm{L}$, indicating that PBDE is a potent neurotoxicant and it might obviously inhibit the development of cultured neurons.

polybrominated diphenyl ether (PBDE), neuron, neurite, synapse, development inhibition

Brominated flame retardants (BFRs), a class of additive flame retardants, have been largely added into industrial products to prevent fires and resulting damages. Polybrominated diphenyl ether (PBDE), constituting an important group of brominated flame retardants, possesses eminent characteristics including high retardant efficiency, thermal stability, less additive amount, and relatively low cost, and accordingly, it has enjoyed widespread use as additives in various manufactured products, such as electronic devices, electrical appliance, chemical polymers, traffic vehicles, building materials, textiles, petroleum, mining substances, etc. ${ }^{[1,2]}$. Widely using PBDE induces more and more serious environmental pollution. Up to now, PBDE has been found in many environmental matrixes and organisms, such as fish, sediment, sewage sludge, human serum, lipid tissues, and breast milk ${ }^{[3-6]}$. PBDE is highly lipophilic, chemically stable, and not readily degraded, and therefore it could bioaccumulate through the food chain up to a high level in the human body, which leads to the potential threat to human health ${ }^{[7-10]}$.

PBDE congeners are non-coplanar owing to the oxygen linkage between the phenyl rings and induce many of the alike toxic effects as structurally similar polychlorinated biphenyl (PCB) and dioxin. The toxicities of PCB and dioxin have been extensively studied; however, the basic toxic actions of PBDEs on organisms are quite limited as PBDEs are a class of emerging pollutants. PBDEs have been found in the brains of mice after exposure to ${ }^{14} \mathrm{C}$-labeled $\mathrm{PBDEs}{ }^{[11]}$. Recent investigations show that the target organs and tissues of PBDE are mainly the adipose tissue, thyroid, reproduction and development, and central nervous system ${ }^{[1,4,8-10]}$. Morris water maze tests suggest that neonatal PBDE exposures at postnatal day 10 to $10.5 \mathrm{mg} / \mathrm{kg}$ of BDE-47 and 12.0

\footnotetext{
Received February 11, 2007; accepted May 23, 2007 doi: 10.1007/s11426-007-0115-8

'Corresponding author (email: gbjiang@rcees.ac.cn)

Supported by the National Basic Research Program of China (Grant No. 2003CB415001), the National Natural Science Foundation of China (Grant No. 30321002) and the Chinese Academy of Sciences (Grant No. KJCX2-SW-H06)
} 
$\mathrm{mg} / \mathrm{kg}$ of BDE-99 induce long-lasting effects in mice, which include aberrations of spontaneous behaviour, that are more pronounced with increasing age, and impairment in learning and memory processes at adulthood. Comparatively, the neurotoxicity of BDE-99 is stronger than that of BDE- $47^{[12]}$. Branchi's study indicated that administration with low doses of PBDEs during critical phases of brain development, such as during the gestation and/or neonatal period in the mouse, has been shown to induce irreversible changes in adult brain function ${ }^{[13]}$. Viberg reported that exposures of diverse PBDE congeners, including BDE-47, BDE-99, BDE153 , and BDE-209, caused similar developmental neurotoxic effects, such as changes in spontaneous behaviors containing locomotion, rearing, and total activity ${ }^{[14]}$. Determination of some protein levels in the striatum and the hippocampus in neonatal mice treated with BDE-99 demonstrated that BDE-99 could affect the cholinergic and catecholaminergic systems ${ }^{[15]}$. However, investigations on neurotoxicity of PBDE are just onset and mainly focus on nervous behaviors, cognitive function, learning and memory, etc. To our knowledge, little is known about toxicological actions of PBDE on neuronal morphology and chemical substance alteration in the brain.

Development of neurons is of great importance in connecting of neural circuit and signalling between neurons. In general, endogenous and extraneous interference, especially extraneously chemical interference, affect the normal neurite development in neurons, and in turn disturb signal transmission. Synapse is an extremely local and special connecting site between two neurons, in which the signal transmission is evoked by a massive neurotransmitters release from the presynaptic terminals of one neuron and subsequent activation of postsynaptic receptors on the dendrites of another neuron. Synaptogenesis occurs in the early period of neuronal development and understanding the influence factors of synaptogenesis significantly contributes to studies regarding physiology and pathology in the brain. In this article, one of the predominantly commercial PBDEs, decabromodiphenyl ether (deca-BDE), was applied to examine effect of PBDE exposure on neurite growth, including neurite length and bifurcation number, and synapse formation and development in cultured hippocampal neuron of rat, based on an approach combining the lipid-mediated transfection and immunostaining. These findings might morphologically contribute to evaluation of PBDE toxicity to the development of neuron.

\section{Material and methods}

\subsection{Cell culture}

Primary hippocampal neuron cultures were made according to the previous reference with some modification $^{[16]}$. E17 to E18 embryonic Sprague-Dawley rats (afforded by the animal facility of Institute of Neuroscience, Chinese Academy of Sciences) were dissected under anaesthesia, and hippocampi were taken out and dissociated with $0.125 \%$ trypsin (Sigma) in the solution of Dubecco's Modified Eagle Medium (DMEM, GIBCO) supplemented with $10 \%$ heat-inactivated fetal bovine serum (GIBCO) and $10 \%$ Ham's F-12 (GIBCO) at $37^{\circ} \mathrm{C}$ for $12 \mathrm{~min}$. The disperse neurons were planted on poly- $D$-lysine-coated (Sigma) glass coverslips at 25000 -60000 cells $/ \mathrm{mL}$ and then incubated in a humid incubator with $95 \%$ air and $5 \% \mathrm{CO}_{2}$. The culture medium was half-changed after $24 \mathrm{~h}$ and then once every week with the maintenance medium containing Neurobasal media (GIBCO), 2\% B-27 supplement and 0.25\% glutamine (GIBCO). Both neurons and glial cells were presented in this culture condition. Excess glial cell proliferation was inhibited by administration of $2-4 \mathrm{mmol} / \mathrm{L}$ of cytosine arabinoside (Sigma) in culture medium at 6 or $7 \mathrm{~d}$ in vitro (DIV).

\subsection{Lipid transfection}

Neurons were transfected with plasmid using Lipofectamine2000 (Invitrogen) according to the manufacturer's protocol. The Opti-MEM medium (GIBCO) without serum and antibiotic agent was used in this process. The lipid and DNA (the ratio of lipid and DNA was $2 \mu \mathrm{L}: 1$ $\mu \mathrm{g}$ ) were respectively added into $250 \mu \mathrm{L}$ of the medium and two solutions were mixed equably 5 min later. After additional $20 \mathrm{~min}$, the mixture medium was transferred to the $\phi 35 \mathrm{~mm}$ of dish with neurons to be transfected, incubated at $37^{\circ} \mathrm{C}$ for $3 \mathrm{~h}$, and then exchanged with the original Neurobasal medium.

\subsection{Measurement of neurite length and bifurcation number}

After planting, neurons were exposed to deca-BDE (Great Lakes Chemical Corporation, IN; 97\%) dissolved in dimethylsulfoxide (DMSO, Sigma) immediately. The exposure media were half-changed every other day to regulate the relatively stable exposure concentrations. 
Neurons were transfected at 4 DIV with green fluorescent protein (GFP) plasmid, fixed in 4\% paraformaldehyde (Merck) for $10 \mathrm{~min}$, washed with $0.1 \mathrm{~mol} / \mathrm{L}$ PBS buffer, and imaged with a Fluoview 500 IX71 confocal laser-scanning microscope (Olympus, Japan). Neurites, comprising axon and dentrite, take part in the connection of neural circuitry, which play a crucial role in signalling between neurons. The neurite length and bifurcation number were summarized using the Image-Pro Plus 5.1 professional image analysis software (Media Cybernetics, Carlsbad, CA). Two parallel treatments were made in each group and the experiment was repeated three times.

\subsection{Immunostaining}

Functional synapses among neurons can be labeled with a marker of postsynaptic density protein 95 (PSD95), and the expression level of dentritic PSD95 in neurons reflects the functional synapse formed ${ }^{[17]}$. Appropriate amount of deca-BDE was added into the medium when neurons planted in dishes. The exposure media were half-changed every other day to regulate the relatively stable exposure concentrations. After 14 or $15 \mathrm{~d}$ of exposure, neurons were fixed in $4 \%$ paraformaldehyde for $10-15 \mathrm{~min}$ at room temperature, permeabilized with $0.2 \%$ Triton X-100 (Sigma) for $10 \mathrm{~min}$, and then washed three times with $0.1 \mathrm{~mol} / \mathrm{L}$ PBS buffer. The neurons were pre-incubated with $10 \%$ bovine serum albumin (GIBCO) for $1 \mathrm{~h}$ at room temperature and then incubated with the primary antibody anti-PSD95 (1:200, Affinity BioReagents, Golden, CO) to block nonspecific binding overnight at $4{ }^{\circ} \mathrm{C}$. After washing to remove excess primary antibodies with PBS saline, the cultures were incubated for $1 \mathrm{~h}$ at room temperature with cy3-conjugated secondary antibodies $(1: 1000$, Molecular Probes, Eugene, OR). Excess antibody was removed, and cells were photographed using the Olympus confocal microscope. Two parallel treatments were made in each group, and the experiment was repeated three times.

\subsection{Statistics}

The morphological photos of neurons were imaged by the Fluoview 500 IX71 confocal microscope and analyzed with Image-Pro Plus 5.1 interface. Data were showed as mean \pm SEM and differences between groups were determined by one-way ANOVA. $P<0.05$ was considered as significant.

\section{Results and discussion}

\subsection{Effect of PBDE on neurite growth}

Representative images photographed by the Olympus confocal microscope were showed in Figure 1. This figure suggested that the solvent group (DMSO group) was comparable with the control group in which neurons showed normal development with long neurites and much bifurcation, indicating that the solvent DMSO had no obvious effects on the neurite growth of cultured neurons in this experiment condition. Otherwise, the morphologies of neurons exposed to $15 \mu \mathrm{mol} / \mathrm{L}$ decaBDE were impaired in different degrees in which neurons showed short neurites and less bifurcation. In this study, the transfected neurons were examined for four features: 1) the length of the longest neurite, irrespective of whether the longest neurite was the axon or not, 2) the sum length of neurites per cell, 3) the number of bifurcated neurites, and 4) the number of neurites extending from the soma. Figure 3 listed the normalized results. Quantitative measurements indicated that $10 \mu \mathrm{mol} / \mathrm{L}$ deca-BDE treatment did not basically alter the lengths of the longest neurites (Figure 3A, $P>0.05$ ). In contrast, $15 \mu \mathrm{mol} / \mathrm{L}$ treatments decreased the lengths about $22 \%$ (Figure 3A, $P<0.05$ ). Summary data regarding to the sum length of neurites per neuron displayed that $10 \mu \mathrm{mol} / \mathrm{L}$ deca-BDE administrations declined the sum lengths around $12 \%$ but not significantly (Figure $3 \mathrm{~B}, P>0.05$ ); $15 \mu \mathrm{mol} / \mathrm{L}$ administrations significantly decreased the sum lengths up to $40 \%$ (Figure 3B, $P<0.001$ ). Summarized data suggested that deca-BDE-induced alteration pattern in the number of bifurcated neurites was similar to that in the sum length of neurites per neuron (Figure $3 \mathrm{C})$. Both 10 and $15 \mu \mathrm{mol} / \mathrm{L}$ deca-BDE poisoning markedly declined the number of neurites extending from the soma (Figure 3D, $P<0.05$ ). These results indicated that PBDE exposure inhibited the development of cultured hippocampal neuron.

Many evidences demonstrate that the normal neurite outgrowth of neuron is dependent on a specific range of intracellular free calcium concentration $\left(\left[\mathrm{Ca}^{2+}\right]_{\mathrm{i}}\right)$, and abnormal and prolonged elevation of $\left[\mathrm{Ca}^{2+}\right]_{\mathrm{i}}$ can inhibit neurite outgrowth in many cell types ${ }^{[18-20]}$. Neurite bifurcation relies on a variety of $\mathrm{Ca}^{2+}$-dependent processes and both attraction and repulsion movements of neurite require modulation of $\left[\mathrm{Ca}^{2+}\right]_{\mathrm{i}}^{[21,22]}$. Our data showed that both the length and bifurcation number of neurites were 


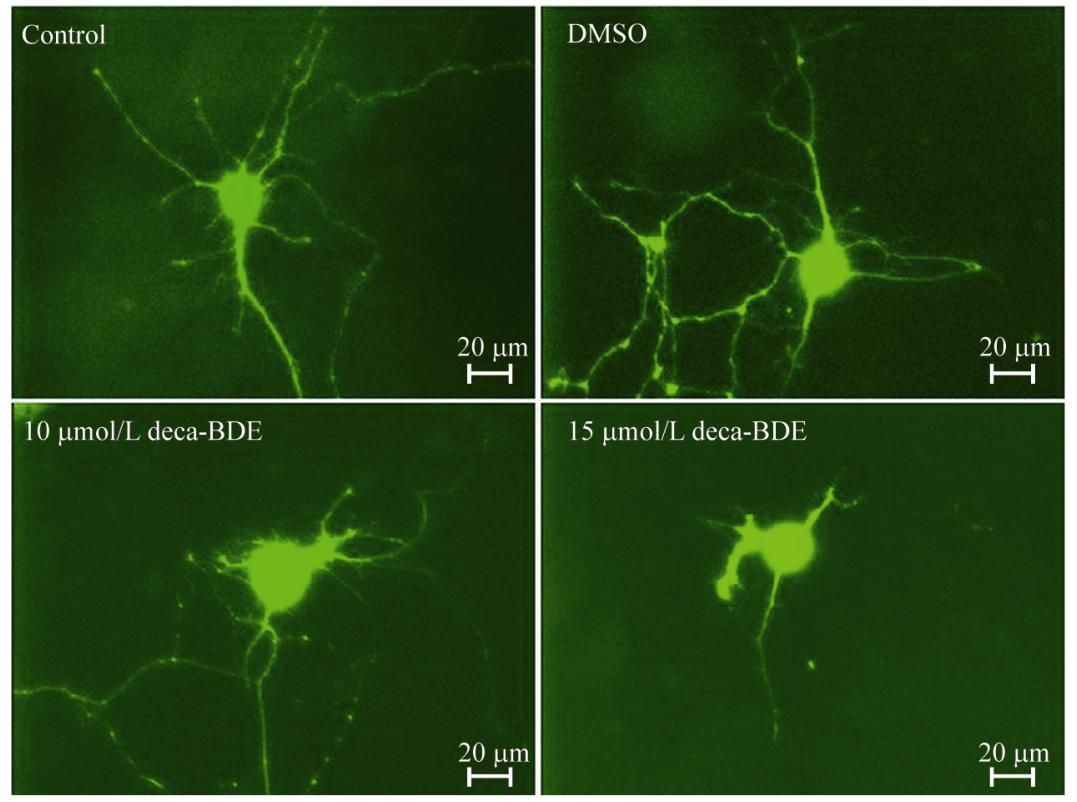

Figure 1 Representative images showed that deca-BDE treatment inhibited the neurite growth in cultured hippocampal neurons of rat.
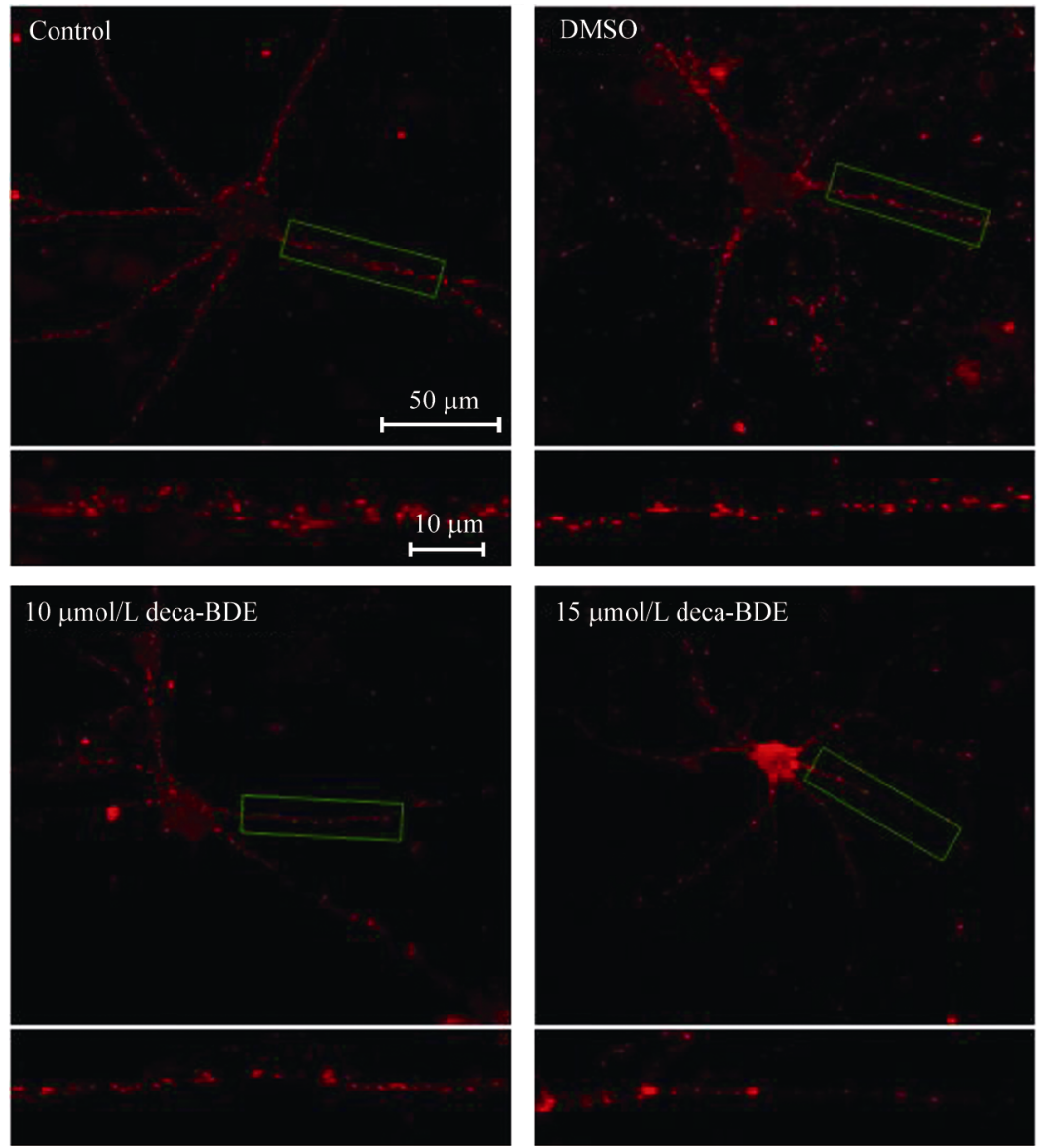

Figure 2 Representative images showed that dendritic PSD95 cluster density in neurons (14 d in vitro) labeled with Cy3 was decreased by deca-BDE exposure. Scale bars, $50 \mu \mathrm{m}$ and $10 \mu \mathrm{m}$ for insets. 
obviously declined by deca-BDE. One possible explanation is that prolonged deca-BDE exposure caused the alteration of $\left[\mathrm{Ca}^{2+}\right]_{\mathrm{i}}$ in neurons, which in turn led to the inhibition of neurite outgrowth. Alm reported that BDE-99 significantly increased levels of neuromodulin (Gap-43) and decreased levels of stathmin in brain. The expression of neuromodulin and stathmin closely correlates with the neurite outgrowth ${ }^{[15]}$. Further experiments need to be carried out to address whether the inhibition of neurite growth by deca-BDE treatment correlated with this process or not.

\subsection{Effect of PBDE on synaptogenesis}

The effect of deca-BDE on expression level of PSD95 in neurons was next examined. As shown in representative images, no significant difference in the level of PSD95 was found between the control and solvent groups. After 14 or $15 \mathrm{~d}$ of deca-BDE exposure, the level of PSD95 in dentrite was markedly decreased (Figure 2). Figure 4 showing the relevant statistic data indicated that 10 and $15 \mu \mathrm{mol} / \mathrm{L}$ deca-BDE administrations declined the level of PSD95 about $21 \%$ and $38 \%$, respectively. Synaptogenesis in the central nervous system comprises the growth and bifurcation of neurite and plays a critical role in neuronal differentiation and circuitry establishment, which is generally interfered by both genetics and environmental factors ${ }^{[23]}$. Here the number of synapses formed was obviously decreased by deca-BDE, which is consistent with the inhibition of neurite growth by deca-BDE as mentioned above. This finding implied that the number of functional synapses was significantly declined after deca-BDE exposure, which will disturb normal synaptic transmission and signalling among neurons.

Evidences are emerging that PBDE are toxic to organisms and the toxicity differs from congener to congener ${ }^{[2,4]}$. Lower brominated ones (tetra- through hexa-BDEs) are more readily absorbed than the higher brominated congeners (hepta- through deca-BDEs). The lower brominated congeners appear to distribute preferentially to lipid rich tissues, especially adipose tissues ${ }^{[4,5,8]}$. The studies of PBDE exposure in rodents in
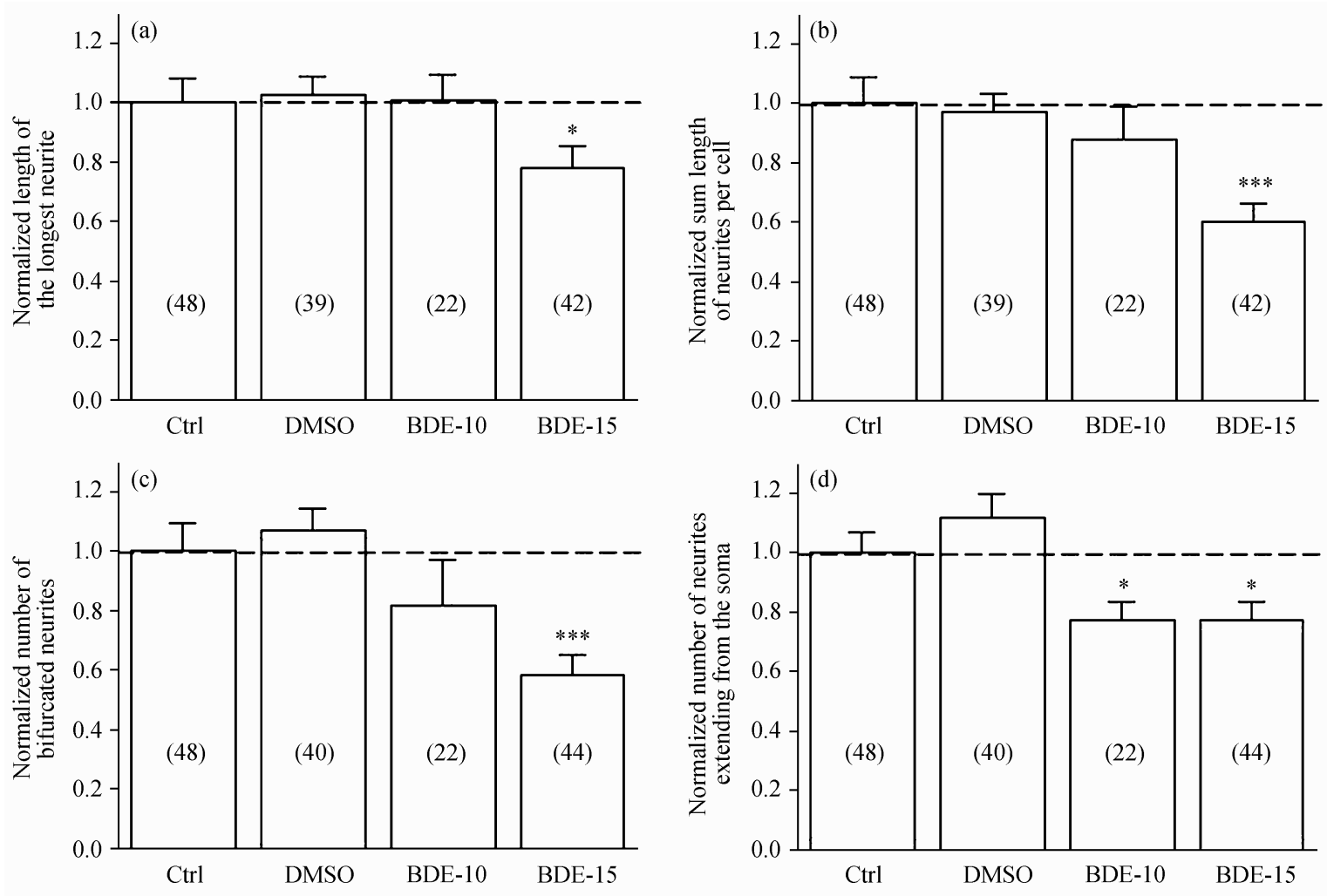

Figure 3 Summary results showed development inhibition of neurite in cultured hippocampal neurons of rat by PBDE exposure: (a) Normalized length of the longest neurite; (b) Normalized sum length of neurites per cell; (c) Normalized number of bifurcated neurites; (d) Normalized number of neurites extending from the soma. "BDE-10" and "BDE-15" associated with the X-axis refer to the 10 and $15 \mu \mathrm{mol} / \mathrm{L}$ deca-BDE exposures, respectively. The number in parenthesis refers to the number of cells, from at least three independent experiments, tested in each condition. $* P<0.05, * * * P<0.001$ vs. control, one-way ANOVA. 


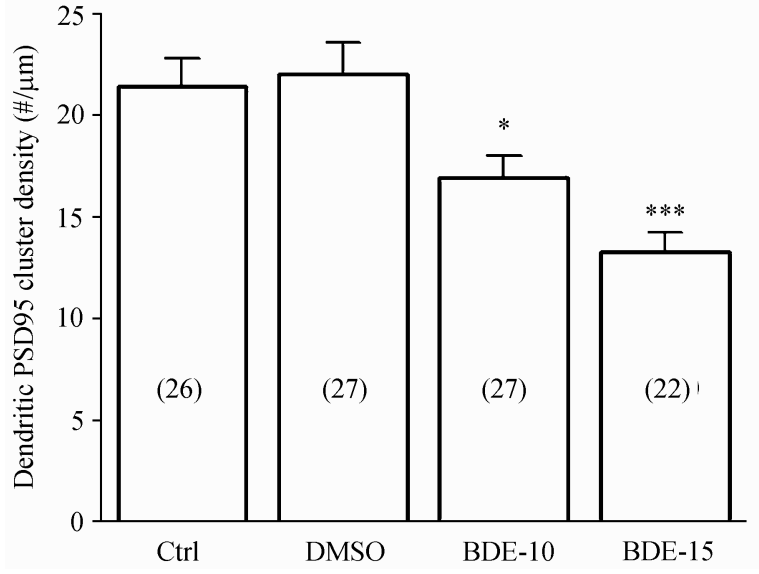

Figure 4 Summarized results showed decrease in dendritic PSD95 cluster density in cultured hippocampal neurons of rat by PBDE exposure. "BDE-10" and "BDE-15" associated with the X-axis refer to the 10 and $15 \mu \mathrm{mol} / \mathrm{L}$ deca-BDE exposures, respectively. The number in parenthesis refers to the number of cells, from at least three independent experiments, tested in each condition. $* P<0.05, * * * P<0.001$ vs. control, one-way ANOVA.

dicated that the neurotoxicity of PBDE could be resulted from alterations in neurotransmitter systems, which may be mediated through the thyroid system ${ }^{[4]}$. Zhou reported that thyroid hormone levels were reduced by several tetra- through octa-BDEs in rats. No alteration of thyroid hormone levels, however, was observed in rats by treatment of deca-BDE with doses as high as 100 $\mathrm{mg} \cdot \mathrm{kg}^{-1} \cdot \mathrm{d}^{-1}$ for four days ${ }^{[24]}$. Deca-BDE can be photodegraded in ultraviolet (UV) light and sunlight to lower brominated $\mathrm{PBDEs}^{[25]}$. Furthermore, deca-BDE is the most predominantly commercial PBDE and it is annually produced throughout the world in great amounts. PBDEs appear to be released in different content to environment from every step of its production, usage, and disposition $^{[26]}$. Therefore, the environmental pollution of deca-BDE should seriously raise our concern. Although the toxicity of deca-BDE is comparatively low, it poses a potential threat to animals and humans due in part to

1 Branchi I, Capone F, Alleva E, Costa L G. Polybrominated diphenyl ethers: Neurobehavioral effects following developmental exposure. Neurotoxicology, 2003, 24: 449-462

2 Liu H X, Zhang Q H, Jiang G B, Cai Z W. Polybrominated diphenyl ethers and its related environmental problems. Prog Chem (in Chinese), 2005, 17(3): 554-562

3 de Wit C A. An overview of brominated flame retardants in the environment. Chemosphere, 2002, 46: 583-624

4 McDonald T A. A perspective on the potential health risks of PBDEs. Chemosphere, 2002, 46: 745-755 its widespread application and possible debromination to lower brominated PBDEs with higher toxicity.

PBDE congeners have been detected in almost all environmental samples from aquatic to terrestrial ecosystems. Frequent PBDE congeners in humans are BDE-47, $99,100,153,154$, and 183, while the most predominantly congeners in workers at dismantling plants of electronic devices are higher brominated BDE-183 and 209 in which their mean PBDE serum concentration was of $37 \mathrm{pmol} / \mathrm{g}$ lipid weight (assumably equal to 37 $\mathrm{nmol} / \mathrm{L})^{[1]}$. Our data with regard to cultured neurons exposed to deca-BDE implied that the lower level of deca-BDE $(10 \mu \mathrm{mol} / \mathrm{L})$ appeared to have little effect on neurite growth and PSD95 clustering density in neurons, which suggests that PBDE possesses relatively low toxicity as compared to other persistent organic pollutants ${ }^{[4,5,8]}$. Considering the remained PBDE serum level $(37 \mathrm{nmol} / \mathrm{L})$ in population occupationally exposed to PBDEs, the value is by far less than $10 \mu \mathrm{mol} / \mathrm{L}$ that posed interference to neurons, which implies that the threat of PBDE to human health is not so severe. Nevertheless, much concern needs to be paid to neurotoxic investigations of deca-BDE because of its bioaccumulation in biota and photodegradation to lower brominated congeners with higher toxicity. Further, morphological alterations in cultured neurons became evident at 15 $\mu \mathrm{mol} / \mathrm{L}$ of deca-BDE exposure, which actually confirmed impairment of PBDE to neuronal development and function.

\section{Conclusion}

PBDE exposures decreased the neurite length and bifurcation number and dendritic PSD95 cluster density in cultured rat hippocampal neurons, which demonstrated that PBDE can significantly inhibit neuronal development, including the growth and branching of neurites, and formation and functions of central synapses.

5 Darnerud P O, Eriksen G S, Jóhannesson T, Larsen P B, Viluksela M. Polybrominated diphenyl ethers: Occurrence, dietary exposure, and toxicology. Environ Health Perspect, 2001, 109(Suppl 1): 49-68

6 Schecter A, Pavuk M, Päpke O, Ryan J J, Birnbaum L, Rosen R. Polybrominated diphenyl ethers (PBDEs) in U.S. mothers' milk. Environ Health Perspect, 2003, 111: 1723-1729

7 Chen G S, Konstantinov A D, Chittim B G, Joyce E M, Bols N C, Bunce N J. Synthesis of polybrominated diphenyl ethers and their capacity to induce CYP1A by the Ah receptor mediated pathway. Environ Sci Technol, 2001, 35: 3749-3756 
8 Darnerud P O. Toxic effects of brominated flame retardants in man and in wildlife. Environ Int, 2003, 29: 841-853

9 Viberg H, Fredriksson A, Jakobsson E, Örn U, Eriksson P. Neurobehavioral derangements in adult mice receiving decabrominated diphenyl ether (PBDE 209) during a defined period of neonatal brain development. Toxicol Sci, 2003, 76: 112-120

10 Mundy W R, Freudenrich T M, Crofton K M, DeVito M J. Accumulation of PBDE-47 in primary cultures of rat neocortical cells. Toxicol Sci, 2004, 82: 164- 169

11 Darnerud P O, Risberg S. Tissue localisation of tetra- and pentabromodiphenyl ether congeners (BDE-47, -85 and -99) in perinatal and adult C57BL mice. Chemosphere, 2006, 62: 485-493

12 Eriksson P, Jakobsson E, Fredriksson A. Brominated flame retardants: A novel class of developmental neurotoxicants in our environment? Environ Health Perspect, 2001, 109: 903-908

13 Branchi I, Alleva E, Costa L G. Effects of perinatal exposure to a polybrominated diphenyl ether (PBDE-99) on mouse neurobehavioural development. Neurotoxicology, 2002, 23: 375-384

14 Viberg H, Fredriksson A, Eriksson P. Investigations of strain and/or gender differences in developmental neurotoxic effects of polybrominated diphenyl ethers in mice. Toxicol Sci, 2004, 81: 344-353

15 Alm H, Scholz B, Fischer C, Kultima K, Viberg H, Eriksson P, Dencker L, Stigson M. Proteomic evaluation of neonatal exposure to 2,2',4,4',5-pentabromodiphenyl ether. Environ Health Perspect, 2006, 114: $254-259$

16 Li C, Lu J, Wu J, Duan S, Poo M. Bidirectional modification of presynaptic neuronal excitability accompanying spike timing-dependent synaptic plasticity. Neuron, 2004, 41: 257-268

17 van Zundert B, Yoshii A, Constantine-Paton M. Receptor compart- mentalization and trafficking at glutamate synapses: a developmental proposal. Trends Neurosci, 2004, 27: 428-437

18 Acebes A, Ferrús A. Cellular and molecular features of axon collaterals and dendrites. Trends Neurosci, 2000, 23: 557-65

19 Kater S B, Mattson M P, Cohan C, Connor J. Calcium regulation of the neuronal growth cone. Trends Neurosci, 1988, 11: 315-321

20 Webb B, Walker D W, Heaton M B. Nerve growth factor and chronic ethanol treatment alter calcium homeostasis in developing rat septal neurons. Dev Brain Res, 2003, 143: 57-71

21 Fields R D, Neale E A, Nelson P G. Effects of patterned electrical activity on neurite outgrowth from mouse sensory neurons. J Neurosci, 1990, 10: 2950-2964

22 Gómez T M, Snow D M, Letourneau P C. Characterization of spontaneous calcium transients in nerve growth cones and their effect on growth cone migration. Neuron, 1995, 14: 1233-1246

23 Zito K, Svoboda K. Activity-dependent synaptogenesis in the adult Mammalian cortex. Neuron, 2002, 35: 1015-1017

24 Zhou T, Ross D G, DeVito M J, Crofton K M. Effects of short-term in vivo exposure to polybrominated diphenyl ethers on thyroid hormones and hepatic enzyme activities in weanling rats. Toxicol Sci, 2001, 61(1): $76-82$

25 Söderström G, Sellström U, de Wit C, Tysklind M. Photolytic debromination of decabromodiphenyl ether (BDE 209). Environ Sci Technol, 2004, 38: 127-132

26 Alcock R E, Sweetman A J, Prevedouros K, Jones K C. Understanding levels and trends of BDE-47 in the UK and North America: An assessment of principal reservoirs and source inputs. Environ Int, 2003, 29: $691-698$ 